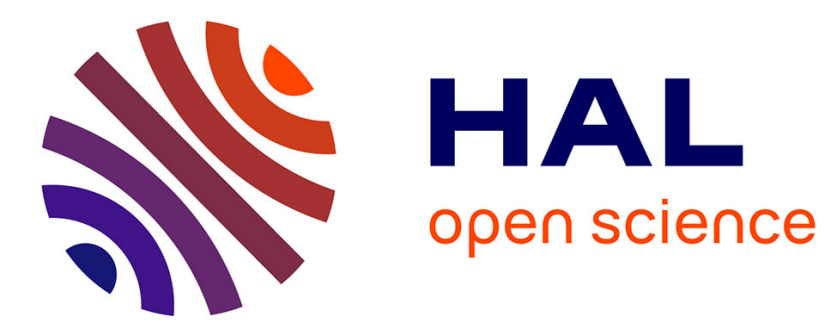

\title{
Solute strongly impacts freezing under confinement
}

Félix Ginot, Théo Lenavetier, Dmytro Dedovets, Sylvain Deville

\section{To cite this version:}

Félix Ginot, Théo Lenavetier, Dmytro Dedovets, Sylvain Deville. Solute strongly impacts freezing under confinement. Applied Physics Letters, 2020, 116 (25), pp.253701. 10.1063/5.0008925 . hal02899319

\section{HAL Id: hal-02899319 \\ https://hal.science/hal-02899319}

Submitted on 8 Oct 2021

HAL is a multi-disciplinary open access archive for the deposit and dissemination of scientific research documents, whether they are published or not. The documents may come from teaching and research institutions in France or abroad, or from public or private research centers.
L'archive ouverte pluridisciplinaire HAL, est destinée au dépôt et à la diffusion de documents scientifiques de niveau recherche, publiés ou non, émanant des établissements d'enseignement et de recherche français ou étrangers, des laboratoires publics ou privés. 


\section{Solute strongly impacts freezing under confinement}

Félix Ginot, ${ }^{1,2}$ Théo Lenavetier, ${ }^{1}$ Dmytro Dedovets, ${ }^{1,3}$ and Sylvain Deville ${ }^{1,4}$

${ }^{1)}$ LSFC, UMR 3080 CNRS/Saint-Gobain CREE, Saint-Gobain Research Provence, 84300 Cavaillon,

France

${ }^{2)}$ now with: Fachbereich Physik, Universität Konstanz, Universitätsstrasse 10, 78464 Konstanz, Germany

${ }^{3)}$ now with: LOF, UMR 5258 CNRS/Solvay, Univ. Bordeaux, 33600 Pessac, France

4) now with: Université de Lyon, Université Claude Bernard Lyon 1, CNRS, Institut Lumière Matière, 69622 Villeurbanne, France

(Dated: 8 October 2021)

The presence of liquid water in frozen media impacts the strength of soils, the growth of frost heave, plant life and microbial activities, or the durability of infrastructures in cold regions. If the effect of confinement alone on freezing is well known, water is never pure and solutes depressing the freezing point are naturally found. However, the combination of confinement and solute is poorly understood. Here we study in-situ the freezing of water in a model porous medium made of densely packed particles with various salt $(\mathrm{KCl})$ concentrations. We demonstrate a synergistic effect of solute with confinement: the freezing front, initially heterogeneous due to confinement, drives solute enrichment in the remaining liquid, further depressing its freezing point. This increases the local freezing point depression and results in much larger mushy layers-where ice and liquid water coexist. We compare our experimental freezing profile with theory, and estimate the local solute concentration to increase by more than one order of magnitude through the freezing process. These results imply that even low solute concentrations may have important effects on the distribution of water in frozen porous media, and should help explain the variety of freezing patterns observed experimentally. This may be critical for cryo-tolerance of construction materials and organisms, and will help understanding solute precipitation and redistribution in soils.

As temperature is lowered below $0^{\circ} \mathrm{C}$, water in porous media can stay partially frozen, which impacts their physical and mechanical properties ${ }^{1}$. In cold regions, frost heaving damages infrastructures such as roads, train tracks, pipelines, and buildings ${ }^{2}$. It is also a major issue for pollution confinement, where soils can be frozen to decrease their permeability ${ }^{3}$. More surprising, it could explain the fluidized transport of sediments on Mars ${ }^{4}$. Understanding the freezing of water in confined media is thus essential in many domains, from cryopreservation to freeze-drying of food, geophysics, and civil engineering.

For pure water, adsorption and interfacial curvature effects $^{5}$ lead to freezing point depression, which can be as low as several tens of degrees in nm- or $\AA$-size pores ${ }^{6}$. Water is however rarely pure and impurities such as ions or organic matter are usually present. The ions $\mathrm{Ca}^{2+}$, $\left.\mathrm{K}^{+}, \mathrm{Na}^{+}\right)$commonly encountered in soils or constructions materials (because of de-icing) are well-known for depressing the freezing point of water. As ice is formed pure, all solutes are expelled and concentrated near the growing ice. This not only further depresses the freezing point, but also creates gradients of solute.

The accumulation of solute may result in precipitation and impact plant life and microbial activities ${ }^{7}$, while the presence of liquid pockets and films weakens the strength of the soil ${ }^{1}$ and provides pathways for water motion and solute redistribution through the soils or sea ice ${ }^{8}$. Although the migration of solutes has been investigated in soil physics ${ }^{9}$ and microbiology ${ }^{10}$, observations and understanding of the synergistic effects of confinement and solute at a local scale are still missing. To date, stud- ies have mostly focused on measuring the relationship between the freezing point and the solute content ${ }^{11}$, assessing the macroscopic redistribution of solutes in soils during ${ }^{12}$ or after freeze/thaw cycles ${ }^{13}$, its crystallization in pores ${ }^{14}$, and quantifying the amount of unfrozen water in soils by NMR or calorimetry ${ }^{15-17}$. Here we show by in-situ, local observations, that the combined effect of confinement and solute during freezing is more than the sum of individual contributions and that this synergy is explained by the interplay between the local thermodynamics of freezing and solute rejection and concentration at the pore scale.

We investigate confined freezing by confocal microscopy $^{18}$, translating a $100 \mu \mathrm{m}$ thick Hele-Shaw cell enclosing a porous medium made of randomly packed monodispersed particles $(2 \mu \mathrm{m}$ polymethyl methacrylate). The sample was centrifuged at a $10 \mathrm{~g}$ gravity for 5 min to achieve a more compact packing of the particles. The sample is moved at a constant velocity $V=2 \mu \mathrm{ms}^{-1}$ through a one-dimensional temperature gradient $\nabla T=10 \mathrm{~K} \mathrm{~mm}^{-1}$ (Fig. $1 \mathrm{~b}$, see SI for more information). The system was designed with a cover on top of the sample to shield from the thermal mass of the objective. We verified in previous work on transparent systems (emulsions) ${ }^{18,19}$ that the temperature gradient through the thickness of the sample is negligible. We record the freezing process with two photodetectors: one to track the fluorescent particles, the other to detect a fluorescent dye (sulforhodamine 101) added to water and expelled by the growing ice. We thus follow in-situ the freezing process through the confined media. 
(a)

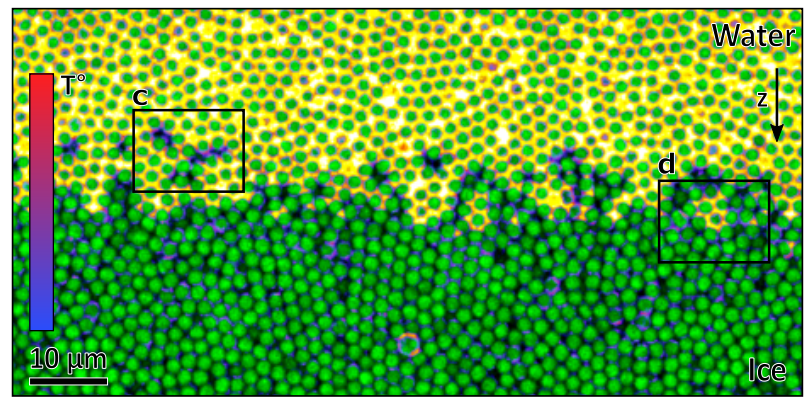

(b)

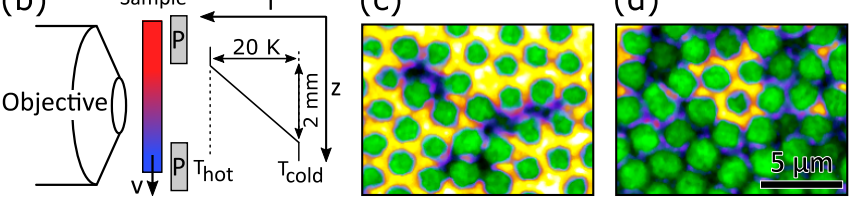

FIG. 1. Experimental setup. (a) Typical picture of freezing into a porous media made of randomly close-packed fluorescent particles (cropped and enhanced). The porous media is shown in green, and liquid water in yellow. (b) Sketch of the freezing setup. Two Peltier modules provide a controlled temperature gradient $\nabla T=10 \mathrm{~K} \mathrm{~mm}^{-1}$. The sample is moved at a constant velocity $v=2 \mu \mathrm{ms}^{-1}$. (c) Close-up view of an isolated region where ice first appears. (d) Closeup view of an isolated liquid region within a frozen shell.

A typical confocal image is shown in Fig. 1a. The propagation of freezing (movie S1) does not occur homogeneously. Not only does the freezing front not follow isotherms - its boundary is convoluted - but we also observe isolated liquid and frozen pockets: ice and liquid water can coexist at the same temperature. Isolated regions that freeze at a higher temperature than the rest of the porous matrix seem to correspond to larger pores (Fig. 1c). Conversely, the densely packed regions (small pores) seem to freeze last (Fig. 1d). In absence of solute, confinement alone can explain this behavior. The freezing-point depression induced by confinement originates from the Gibbs-Thomson effect ${ }^{20}$, where the ice/water interface curvature affects the bulk free energy of the system. In a porous media, the freezing point depression $\Delta T_{f}$ writes:

$$
\Delta T_{f}=T_{m}-T_{f}=\frac{k_{g} T_{m} \gamma_{i w}}{\mathrm{R} \rho_{i} \Delta H_{f}},
$$

where $T_{m}=273 \mathrm{~K}, \rho_{i}=0.92 \mathrm{~g} \mathrm{~cm}^{-3}, \gamma_{i w}=29 \mathrm{~mJ} \mathrm{~m}^{-2}$, $\Delta H_{f}=333 \mathrm{~J} \mathrm{~g}^{-1}$, and $\mathrm{R}$; are the bulk melting temperature, the ice density, the ice/water surface tension, the bulk enthalpy of fusion, and the pore radius respectively. $k_{g}$ is a geometric prefactor that depends on the porous structure and the wetting properties.

As we do not measure the absolute local temperature, we define an undercooling $\mathrm{U}$. By convention $\mathrm{U}=0$ at the beginning of the freezing process, and increases when the local temperature decreases (see SI). From our image analysis, we compute the probability for water to be frozen, for a given pore size $\mathrm{R}$ and undercooling $\mathrm{U}$ (a)

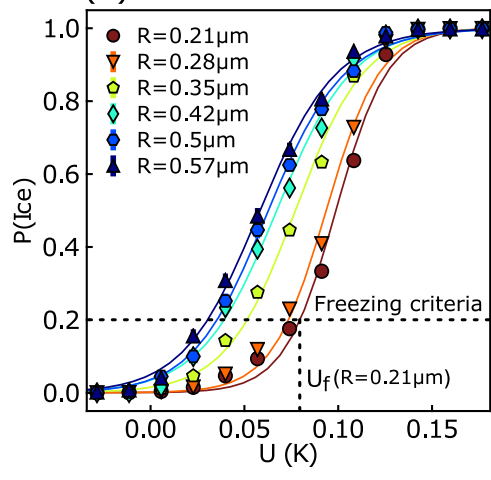

(b)

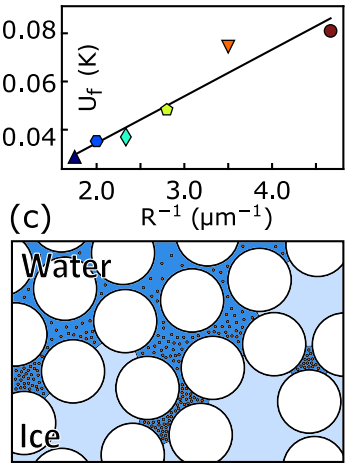

FIG. 2. Gibbs-Thomson Analysis. (a) Probability to find ice for increasing pore size $\mathrm{R}$ as a function of undercooling $\mathrm{U}$. Lines correspond to the interpolation of the experimental data points (see SI) (b) Freezing undercooling $\mathrm{U}_{\mathrm{f}}$ as a function of the inverse of pore size $R^{-1}$. (c) Schematic representation of confined freezing and the concentration of the excluded solute in the remaining liquid. Red dots represent solute in solution.

(Fig. 2a). As expected, ice is more likely found in bigger pores, and at higher undercooling (lower temperature). Between the largest $(\mathrm{R}=0.57 \mu \mathrm{m})$ and the smallest $(\mathrm{R}=0.21 \mu \mathrm{m})$ pores, we find that confinement results in a $\sim 0.05 \mathrm{~K}$ freezing temperature depression.

Note that in the absence of kinetics related effectsand perfectly defined pore size - we expect a sharp freezing transition. Here we find that this transition (that we attribute to kinetics) occurs over $\sim 0.1 \mathrm{~K}$. For a given pore size $\mathrm{R}$, we define the freezing undercooling $\mathrm{U}_{\mathrm{f}}(R)=\mathrm{U}(R, \mathrm{P}($ Ice $)=0.2)$ when $20 \%$ of the water is frozen. We verify the Gibbs-Thomson relationship by plotting $U_{f}$ as a function of the inverse pore size $R^{-1}$ (Fig. 2b). As expected from Eq. 1, a linear relationship is observed, and we obtain $k_{g}=0.73$. While this value is arguably lower than $k_{g}^{c y l}=2$ for cylindrical pores and perfect wetting, we made three hypotheses in this model. First, we only have access to the surface of the sample, while the freezing actually happens in 3D (100 um thick sample). However, the analysis is only performed on the pores we can image in the focal plane. Then, we used a $2 \mathrm{D}$ pore size measurement and neglected any connection bottleneck between the pores. This hypothesis is reasonable as the 3D interconnection is expected to be of same size as the 2D porosity, and we do not expect connection bottlenecks in a random close packing arrangement of spherical particles. Finally, we assumed a perfect wetting between colloids and water. This last point has a great influence on the freezing-point depression and can even result in negative $k_{g}$ in exotic systems ${ }^{21}$. Overall this agreement with theory (linear dependancy and single prefactor value) suggests that the freezing point depression measured here is indeed due to confinement, and thus validate our analysis.

Having validated the approach for the case of confined freezing, we now look at the impact of solute. As 
(b)
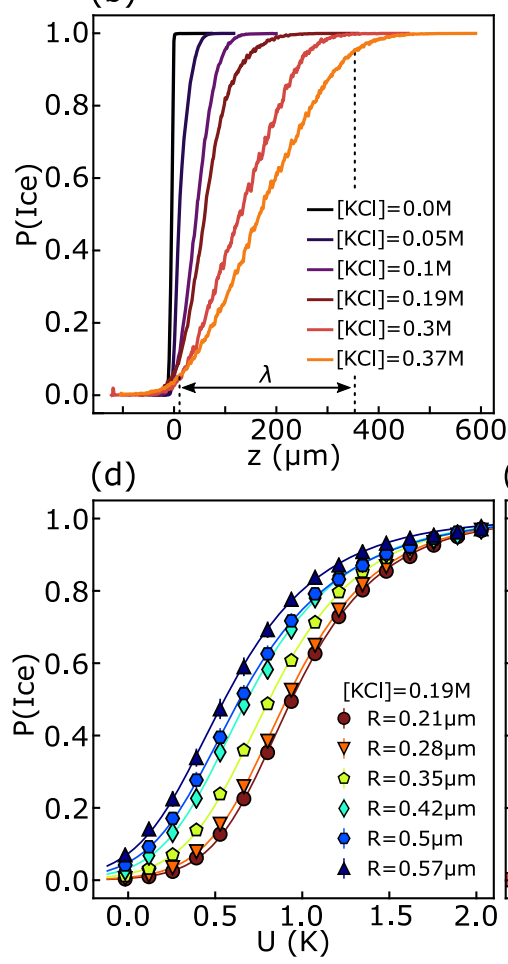

(a)
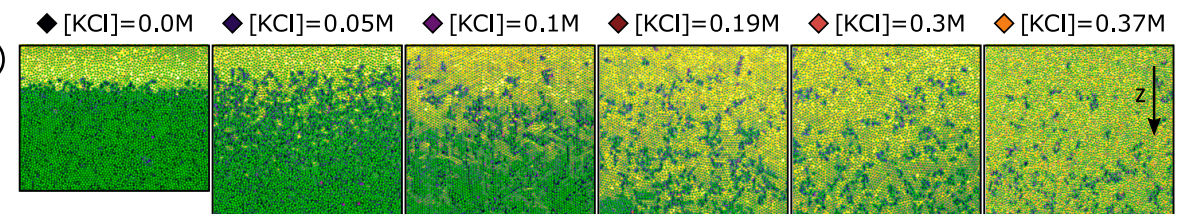

(c)

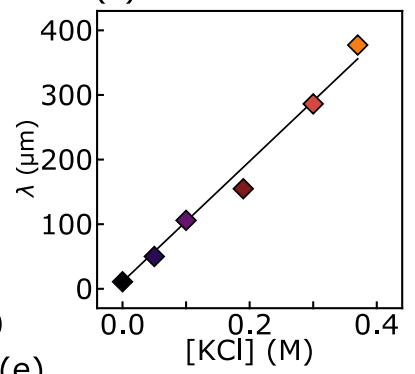

(e)
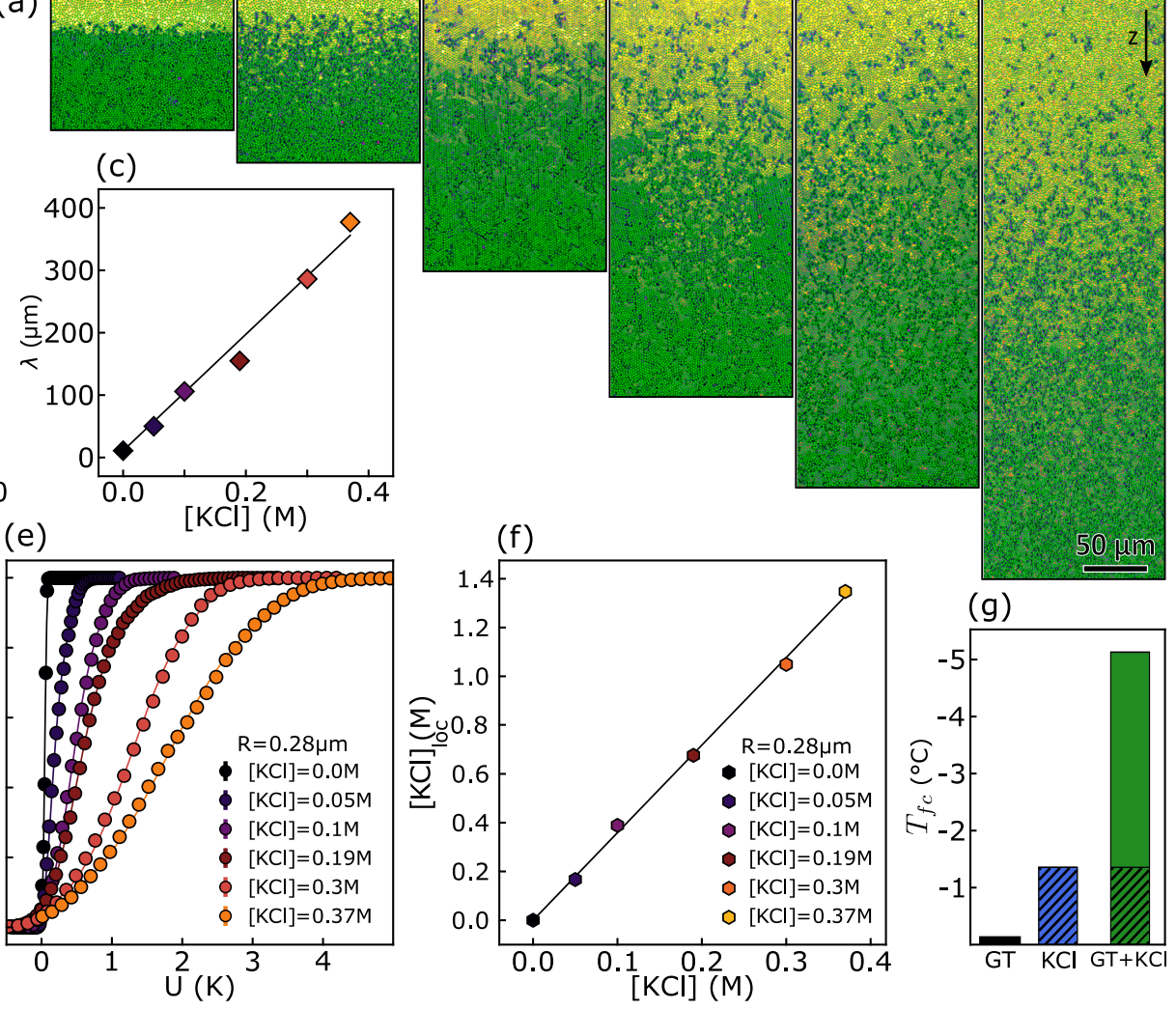

(g)

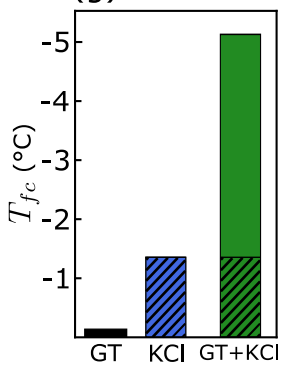

FIG. 3. Solute-Confinement synergy. (a) Confocal images of the mushy layer for increasing solute concentrations (cropped and enhanced). (b) Probability to find ice as a function z position, and for increasing solute concentration [KCl] from $0 \mathrm{M}$ to 0.37 M. (c) Extension of the mushy layer thickness $\lambda$ increasing linearly with [KCl] concentrations. (d) Probability to find ice as a function of undercooling $\mathrm{U}$ and increasing pore size $([\mathrm{KCl}]=0.19 \mathrm{M})$. (e) Probability to find ice as a function of undercooling $\mathrm{U}$ for increasing $[\mathrm{KCl}]$ concentration $(\mathrm{R}=0.28 \mu \mathrm{m})$. (f) Local concentration $[\mathrm{KCl}]_{\text {loc }}$, estimated at the end of the freezing process (95\% frozen), in pores of size $\mathrm{R}=0.28 \mu \mathrm{m}$, and for different initial solute concentrations [KCl]. For this pore size, we report a three-fold increase of $[\mathrm{KCl}]_{\text {loc }}$ during the freezing process. (g) Freezing completion temperature $T_{f c}$ (temperature at which $95 \%$ of the water is frozen) for confinement alone (Gibbs-Thomson effect), solute alone, and confinement with solute $([\mathrm{KCl}]=0.37 \mathrm{M})$. The hatched areas correspond to the pure solute contribution, and the green plain area to the solute/confinement synergistic effect (see SI)

solutes are generally expelled from ice during freezing, we can expect them to accumulate within liquid pockets surrounded by ice shells (Fig. 2c), affecting the freezing process. We use $\mathrm{KCl}$ as a generic solute, at concentrations commonly encountered in soils ${ }^{22}$. Typical pictures of freezing into the porous media with increasing $[\mathrm{KCl}]$, from $0 \mathrm{M}$ to $0.37 \mathrm{M}$, are shown in Fig. 3a. The probability to find ice as a function of the $z$ position, decreases with the solute concentration (Fig. 3b). As a result, the thickness $\lambda$ of the mushy layer where ice and water coexist (see SI), increases linearly with concentration (Fig. 3c). For the highest concentrations $([\mathrm{KCl}]>0.19 \mathrm{M})$ the freezing front becomes ill-defined, and isolated liquid pockets (and frozen islands) can be observed (see Fig. 3a or Fig. S3).

The depression of the freezing-point of a liquid due to a solute is a phenomenon known since the end of the XVIII $^{t h}$ century (Blagden Law). The addition of a solute to a solvent decreases the chemical potential of the resulting solution and thus its freezing temperature. As a result, for an ideal solution, the freezing temperature decreases linearly with the solute concentration. Here we follow this classical approach (see SI for derivations), and rewrite the Gibbs-Thomson equation adding the solute contribution:

$$
\Delta T_{f}=\frac{k_{g} T_{m} \gamma_{i w}}{\mathrm{R} \rho_{i} \Delta H_{f}}+\frac{2 k_{b} N_{a} T_{m}^{2}}{\Delta H_{f} \rho_{w}}[\mathrm{KCl}],
$$

with $k_{b}$ the Boltzman constant, $N_{a}$ the Avogadro number, and $\rho_{w}=1 \mathrm{~kg} \mathrm{l}^{-1}$ the density of water. The prefactor 2 corresponds to the van 't Hoff factor for $\mathrm{KCl}$.

We reiterate the analysis developed for the solute-free experiments and get a deeper look at the freezing process in the presence of solute. We show in Fig. 3d the probability to find frozen water at a given undercooling $\mathrm{U}$ and different pore sizes R, with $[\mathrm{KCl}]=0.19 \mathrm{M}$. Similar to the solute-free experiments, water in smaller pores freezes later (at lower temperature) than in bigger pores. 
However, the freezing process occurs over a much broader range of temperature. For instance, at $[\mathrm{KCl}]=0.19 \mathrm{M}$, pores of size $\mathrm{R}=0.21 \mu \mathrm{m}$ freeze approximately $0.5 \mathrm{~K}$ after pores of size $\mathrm{R}=0.57 \mu \mathrm{m}$. This temperature gap is one order of magnitude larger than for solute-free experiments $(\sim 0.05 \mathrm{~K})$, and therefore cannot be explained by confinement only.

We now look into the other solute concentrations and try to separate the solute and confinement effects. We show in Fig. 3e the probability for water to be frozen at a given undercooling $\mathrm{U}$, for a fixed pore size $\mathrm{R}=$ $0.28 \mu \mathrm{m}$, and increasing solute concentration $[\mathrm{KCl}](0 \mathrm{M}$ to $0.37 \mathrm{M})$. As expected, solute decreases the probability to find ice for a given undercooling value. Nonetheless, we notice that even for a fixed pore size and initial solute concentration, the freezing process occurs over a broad range of temperature (up to $\Delta \mathrm{U} \sim 4 \mathrm{~K}$ for $[\mathrm{KCl}]=0.37 \mathrm{M})$. The effect is again more than one order of magnitude larger than for solute-free experiments and therefore cannot be explained by freezing kinetics.

These observations can be explained by the accumulation of solute in the porous matrix throughout the freezing process. Initially, the solute concentration is the same in every pore. As freezing proceeds, bigger pores freeze first - due to the Gibbs-Thomson effect - and expel the solute they contained (Fig. 2c). The local solute concentration increases thus as additional pores are freezing. This behavior is best observed in Fig. 3d, where one can clearly see that the differential in freezing undercooling between the smallest and largest pores is much larger than for the solute-free study. Hence, solute and confinement have a synergistic effect: confinement destabilizes the freezing front and allows solute to be expelled, enlarging the mushy layer and delaying the completion of freezing.

Having access to both pore size and freezing point depression, we can use Eq. 2 to estimate the local concentration $[\mathrm{KCl}]_{\text {loc }}$ (see SI). In Fig. 3f, we show $[\mathrm{KCl}]_{\text {loc }}$ at the end of the freezing process (95\% of water are frozen), in pores of size $\mathrm{R}=0.28 \mu \mathrm{m}$. For each experiment with initial concentration $[\mathrm{KCl}]$, we report a three-fold increase of the local $\mathrm{KCl}$ concentration.

In this work we only have access to the micro-porosity (smallest pore size is $\mathrm{R}=0.21 \mathrm{\mu m}$ ) of the system. At this scale, the estimated concentration $(\sim 1.4 \mathrm{M})$ is already approaching the solubility limit $(3.8 \mathrm{M})$ of $\mathrm{KCl}$ in water. As freezing proceeeds in pores increasingly smaller (that we cannot image here), the solute concentration will increase accordingly. In this model porous medium as well as in more realistic systems - with a broader range of porosity - we expect this synergistic process of solute expulsion to reach the nm scale. This could eventually lead to local salt crystallization which can then occur even for low initial concentrations.

Two effects are thus combined in the presence of solute. $\mathrm{KCl}$ depresses the freezing point, which lowers the temperature at which the first (largest) pores will freeze. Once freezing is initiated in the largest pores, the synergy between confinement and solute revealed here will further lower $T_{f c}$, the temperature at which freezing is completed (see SI). These combined effects (Fig. 3g) are more than one order of magnitude greater (up to $\sim 5.14 \mathrm{~K}$ for $[\mathrm{KCl}]=0.37 \mathrm{M})$ than for confinement only $(\sim 0.15 \mathrm{~K})$, and much greater than expected from a simple combination of both effects.

In our experiments, this synergy resulted in large mushy layers spreading over $\sim 100 \mu \mathrm{m}$, even for low solute concentrations. However temperature gradients in soils are much lower $\left(\sim 10 \mathrm{~K} \mathrm{~m}^{-1}\right)$ than in our model system, hence we can expect the mushy layer to reach macro-scales $\left(\lambda_{\text {soil }} \sim 10 \mathrm{~cm}\right)$ in real-life conditions. This could explain the large frozen fringes observed between ice lenses in soils ${ }^{23}$.

The complex morphology of the mushy layer in the presence of salts, with isolated liquid pockets remaining (see Fig. 3a or SI Fig. S3), prevents easy pathways for the salt to diffuse away from these high solute concentration areas (except for premelted films around the particles). We can therefore expect the high salt concentration reached locally to remain and the diffusion of solute away from the concentrated regions is probably limited. This has important consequences in soils physics and plant life, where salinity effects on plants can be critical ${ }^{22}$, and can lead to pitting corrosion in materials such as reinforced concrete $^{24}$.

Finally, after all the micro-porosity of the system has been filled by ice, we anticipate surface charges, polarization forces, and nm-scale effects to become predominant in the system. These length scale are out of the scope of the current study, and difficult to access experimentaly. Nonetheless we can expect premelting to dominate the freezing process, and lead to even larger seggregation effects and local solute concentration ${ }^{25}$. It was also recently suggested ${ }^{26}$ that the rejection of salts ions in confined environment during freezing may result in considerable disjoining pressures at the pore scale, which could explain the freezing-induced damages of construction materials such as concrete. Here we found no effects that could be explained by the developement of a locally large disjoining pressure, nor did we observe any obvious difference in terms of particle organisation with and without solute: the structure remained the same. Due to the nature of the porous medium (random close packing without bonding of adjacent particles) large scale fractures are unlikely to happen. The polymer particles used here likely accomodate, to some extent, any pressure appearing in the sample. This of course may be different in a more porous and/or more rigid medium.

Overall, this study shows that solutes such as salts must be taken into account to interpret the variety of freezing patterns observed experimentally - variety that we still struggle to understand today ${ }^{27}$. The synergy between salt and confinement is explained by the thermodynamics of freezing and solute build-up at the pore scale, and illustrates how small amounts of solute can have a large effect in freezing heterogeneous media such 
as soils or construction materials. Our results provide a practical framework to understand not only the amount and spatial distribution of residual water in frozen media, but also the progressive salt concentration, which is known to greatly impact the cryotolerance properties of construction materials and many organisms. We expect the magnitude of these effects to be enhanced in real life systems, where temperature gradients are much lower, and pore size can be reduced down to nm scale.

\section{SUPPLEMENTARY MATERIAL}

Supplementary Material includes the detailed methods, the derivation of the Gibbs-Thomson equation with the solute contribution, the image analysis procedure, a typical movie of the solidification, and a typical high resolution confocal microscopy image.

\section{ACKNOWLEDGMENTS}

We thank Thomas E. Kodger for providing the particles and Benoit Coasne for the discussion on these results and analyses. Funding: The research leading to these results has received funding from the European Research Council under the European Community's Seventh Framework Programme (FP7/2007-2013) Grant Agreement no. 278004. T.L.'s internship was funded by Saint-Gobain. Author contributions: S.D. designed and supervised the project, S.D. and F.G. designed the experiments, D.D. designed and built the cooling stage, F.G. and T.L. carried out the confocal microscopy, F.G. wrote the code to analyze the data, S.D., F.G. and T.L. analyzed the data. All authors discussed the results and implications. F.G. and S.D. wrote the manuscript. Competing interest: The authors declare no competing interest. Data and materials availability: Additional data and code related to this paper are available on Figshare (10.6084/m9.figshare.12343934).
${ }^{1}$ O. Coussy, J. Mech. Phys. Solids 53, 1689 (2005).

${ }^{2}$ A. W. Rempel, J. Glaciol. 56, 1122 (2010).

${ }^{3}$ I. K. Iskandar, Effect of freezing on the level of contaminants in uncontrolled hazardous waste sites, Tech. Rep. (US Army Corps of Engineer, Special Report 86-19, 1986).

${ }^{4}$ R. Oyarzun, C. Viedma, A. Marrquez, and J. Lillo, Terra Nov. 15, 238 (2003).

${ }^{5}$ J. S. Wettlaufer, Philos. Trans. Ser. A , 3403 (1999).

${ }^{6}$ H. K. Christenson, J. Phys. Condens. Matter 13, R95 (2001).

${ }^{7}$ M. Hayashi, Vadose Zo. J. 12, 2136 (2013).

${ }^{8}$ R. Lake and L. Lewis, J. Geophys. Res. 75, 583 (1970).

${ }^{9}$ K. Henry, CRREL Rep. 88-17, Tech. Rep. (US Army Corps of Engineers, Cold Regions Research and Engineering Laboratory, 1988).

${ }^{10}$ J. Kim, A. H. Lee, and W. Chang, Sci. Total Environ. 612, 903 (2018).

${ }^{11}$ A. Banin and D. M. Anderson, Water Resour. Res. 10, 124 (1974).

${ }^{12}$ K. Watanabe, Y. Muto, and M. Mizoguchi, Cryst. Growth Des. 1, 207 (2001).

${ }^{13}$ J.-M. Konrad and A. W. McCammon, Can. Geotech. J. 67, 726 (1990).

${ }^{14}$ G. W. Scherer, Cem. Concr. Res. 29, 1347 (1999).

${ }^{15}$ K. Watanabe and M. Mizoguchi, Cold Reg. Sci. Technol. 34, 103 (2002).

${ }^{16} \mathrm{~K}$. Watanabe and T. Wake, Cold Reg. Sci. Technol. 59, 34 (2009).

${ }^{17}$ H. Bing and W. Ma, Cold Reg. Sci. Technol. 67, 79 (2011).

${ }^{18}$ D. Dedovets and S. Deville, Journal of the European Ceramic Society 38, 2687 (2018).

${ }^{19}$ D. Dedovets, C. Monteux, and S. Deville, Annu. Rev. Fluid Mech. 38, 427 (2006).

${ }^{20} \mathrm{~J}$. Wettlaufer and M. G. Worster, Annu. Rev. Fluid Mech. 38, 427 (2006).

${ }^{21}$ A. Watanabe, T. Iiyama, and K. Kaneko, Chemical physics letters 305, 71 (1999).

${ }^{22}$ A. K. Parida and A. B. Das, Ecotoxicology and environmental safety 60, 324 (2005).

${ }^{23}$ S. Peppin, A. Majumdar, R. Style, and G. Sander, Siam J. Appl. Math. 71, 1717 (2011).

${ }^{24}$ L. Bertolini, B. Elsener, P. Pedeferri, E. Redaelli, and R. Polder, Corrosion of steel in concrete, Vol. 392 (Wiley Online Library, 2013).

${ }^{25}$ H. Hansen-Goos and J. Wettlaufer, Physical Review E 81, 031604 (2010).

${ }^{26}$ T. Zhou, M. Mirzadeh, R. J.-M. Pellenq, and M. Z. Bazant, arXiv preprint (2019), arXiv:1905.07036v1.

${ }^{27} \mathrm{~J}$. Wettlaufer, Philosophical Transactions of the Royal Society A 377, 20180261 (2019). 